\title{
Research Article \\ On the Nusselt Solution of a Nonisothermal Two-Fluid Inclined Film Flow
}

\author{
Jürgen Socolowsky \\ Mathematics Group, Engineering Department, Brandenburg University of Applied Sciences, \\ P.O. Box 2132, 14737 Brandenburg an der Havel, Germany \\ Correspondence should be addressed to Jürgen Socolowsky, socolowsky@fh-brandenburg.de
}

Received 18 August 2009; Revised 13 November 2009; Accepted 26 November 2009

Recommended by Hans Engler

Nonisothermal viscous two-fluid flows occur in numerous kinds of coating devices. The corresponding mathematical models often represent two-dimensional free boundary value problems for the Navier-Stokes equations or their modifications. In the present paper we are concerned with a particular problem of coupled heat and mass transfer. Marangoni convection is incorporated, too. The solvability of a corresponding stationary problem is discussed. The obtained results generalize previous results for a similar isothermal problem.

Copyright (C) 2009 Jürgen Socolowsky. This is an open access article distributed under the Creative Commons Attribution License, which permits unrestricted use, distribution, and reproduction in any medium, provided the original work is properly cited.

\section{Introduction}

Thermocapillary convection describes a fluid motion driven by surface-tension gradients on a liquid-liquid interface, where these gradients arise from the temperature dependence of interface tension. This type of convection is quite important in several technological and scientific applications; interesting examples may be found in the field of materials science, particularly in coating and solidification processes or in crystal-growth processes (cf. [1-5]).

In this paper we study a problem for a 2D stationary flow with two viscous incompressible heat-conducting fluids (having kinematic viscosities $v_{i}>0$, densities $\rho_{i}>0$, and thermal conductivities $\lambda_{i}, i=1,2$ ) down an inclined bottom $S_{0}$ having a slope $\alpha$ (cf. Figure 1). In fact, the bottom $S_{0}$ represents a perturbed plane. Assume that the bottom is given by the formula $S_{0}=\left\{\mathbf{x}=\left(x_{1}, x_{2}\right) \in \mathbb{R}^{2}: x_{2}=\varepsilon \varphi_{0}\left(x_{1}\right),-\infty<x_{1}<+\infty\right\}$ with $\varphi_{0}$ having a compact support, that is, $\varphi_{0}\left(x_{1}\right) \equiv 0$ for $\left|x_{1}\right| \geq R_{0}>0$, and suppose that the direction $\mathbf{e}_{g}$ of the gravitational force is the vector $\mathbf{e}_{g}=(\sin \alpha,-\cos \alpha)^{T}$ which makes (with respect to the chosen coordinate system) an angle $\alpha^{*}:=\pi / 2-\alpha(0<\alpha \leq \pi / 2)$ with the $x_{1}$-axis. Note that the corresponding problem will be formulated in dimensionless form. The concrete transition to that formulation can be found in [6]. 


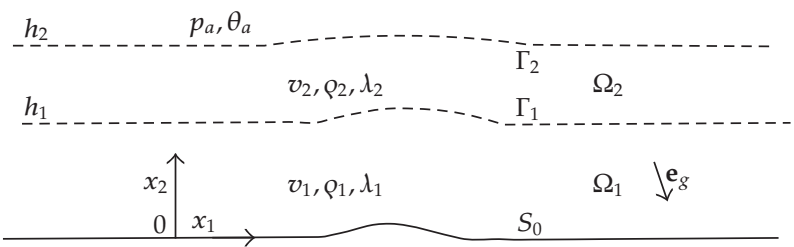

Figure 1: Flow domain of a nonisothermal two-fluid inclined film flow.

Let us formulate the problem. We consider the two-fluid flow down the inclined bottom $S_{0}$ caused by gravity $g \mathbf{e}_{g}$, only. This means mathematically that the positive (final) layer thickness in each liquid layer $\Omega_{i}(i=1,2)$ is a priori prescribed. In slide coaters, such flows occur on some parts of the coater. The corresponding flow fields and layer profiles are essential there.

Suppose that the free interface $\Gamma_{1}$ separating the two fluid layers and the upper free surface $\Gamma_{2}$ admit the parametrizations $\Gamma_{i}=\left\{\mathbf{x} \in \mathbb{R}^{2}: x_{2}=\psi_{i}\left(x_{1}\right),-\infty<x_{1}<+\infty\right\}(i=1,2)$, where the functions $\psi_{i}(i=1,2)$ are a priori unknown and have to be found. Let $h_{i}>0(0<$ $\left.h_{1}<h_{2}\right)$ be the prescribed constant limits of $\psi_{i}\left(x_{1}\right)(i=1,2)$, at infinity. The problem under consideration has the following form: to find a vector of velocity $\mathbf{v}=\left(v_{1}\left(x_{1}, x_{2}\right), v_{2}\left(x_{1}, x_{2}\right)\right)^{T}$, a pressure $p\left(x_{1}, x_{2}\right)$, a temperature $\theta\left(x_{1}, x_{2}\right)$, and functions $\psi_{i}\left(x_{1}\right)(i=1,2)$ satisfying in the domain $\Omega=\Omega_{1} \cup \Omega_{2}$ with $\Omega_{1}=\left\{\mathbf{x} \in \mathbb{R}^{2}: \varepsilon \varphi_{0}\left(x_{1}\right)<x_{2}<\psi_{1}\left(x_{1}\right),-\infty<x_{1}<+\infty\right\}$ and $\Omega_{2}=\left\{\mathrm{x} \in \mathbb{R}^{2}: \psi_{1}\left(x_{1}\right)<x_{2}<\psi_{2}\left(x_{1}\right),-\infty<x_{1}<+\infty\right\}$, then the following equations of a coupled heat and mass transfer are

$$
\begin{gathered}
(\mathbf{v} \cdot \nabla) \mathbf{v}-v \nabla^{2} \mathbf{v}+\frac{1}{\rho} \nabla p=g \mathbf{e}_{g}, \\
\nabla \cdot \mathbf{v}=0, \\
(\mathbf{v} \cdot \nabla) \theta-\lambda \nabla^{2} \theta=0,
\end{gathered}
$$

and the boundary and integral conditions are

$$
\begin{gathered}
\left.\mathbf{v}\right|_{S_{0}}=\mathbf{0},\left.\quad \theta\right|_{S_{0}}=\theta_{0}, \\
{\left.[\theta]\right|_{\Gamma_{1}}=0,\left.\quad\left[\lambda \frac{\partial \theta}{\partial n}\right]\right|_{\Gamma_{1}}=0,\left.\quad[\mathbf{v}]\right|_{\Gamma_{1}}=\mathbf{0},} \\
\left.\mathbf{v} \cdot \mathbf{n}\right|_{\Gamma_{1}^{-}}=0,\left.\quad[\boldsymbol{\tau} \cdot \mathbf{S}(\mathbf{v}) \mathbf{n}]\right|_{\Gamma_{1}}=-\left.b_{1} \frac{\partial \theta}{\partial \tau}\right|_{\Gamma_{1}^{-}} \\
\frac{\mathrm{d}}{\mathrm{d} x_{1}} \frac{\psi_{1}^{\prime}\left(x_{1}\right)}{\sqrt{1+\psi_{1}^{\prime}\left(x_{1}\right)^{2}}}=\left.\frac{1}{\sigma_{1}(\theta)}[-p+\mathbf{n} \cdot \mathbf{S}(\mathbf{v}) \mathbf{n}]\right|_{\Gamma_{1}}, \\
\lim _{\left|x_{1}\right| \rightarrow+\infty} \psi_{1}\left(x_{1}\right)=h_{1},
\end{gathered}
$$




$$
\begin{gathered}
\left.\left(\theta-\theta_{a}+\lambda_{2} \frac{\partial \theta}{\partial n}\right)\right|_{\Gamma_{2}}=0, \\
\left.\mathbf{v} \cdot \mathbf{n}\right|_{\Gamma_{2}}=0,\left.\quad \boldsymbol{\tau} \cdot \mathbf{S}(\mathbf{v}) \mathbf{n}\right|_{\Gamma_{2}}=-\left.b_{2} \frac{\partial \theta}{\partial \tau}\right|_{\Gamma_{2}}, \\
\frac{\mathrm{d}}{\mathrm{d} x_{1}} \frac{\psi_{2}^{\prime}\left(x_{1}\right)}{\sqrt{1+\psi_{2}^{\prime}\left(x_{1}\right)^{2}}}=\left.\frac{1}{\sigma_{2}(\theta)}\left(p_{a}-p+\mathbf{n} \cdot \mathbf{S}(\mathbf{v}) \mathbf{n}\right)\right|_{\Gamma_{2}}{ }^{\prime} \\
\lim _{\left|x_{1}\right| \rightarrow+\infty} \psi_{2}\left(x_{1}\right)=h_{2} .
\end{gathered}
$$

In [1] it was shown that for a large number of liquids the surface tensions $\sigma_{i}$ can be regarded as linear functions of the temperature $\theta$ along the free interface $\Gamma_{i}(i=1,2)$ (cf. also $\left.[3,5]\right)$ as follows:

$$
\sigma_{i}(\theta)=a_{i}-b_{i} \theta \quad\left(a_{i}, b_{i}>0, i=1,2\right)
$$

By $\lambda_{m}$ we denote the thermal conductivity of the $m$ th fluid $(m=1,2)$ in Problem (1.1)(1.4). The symbol $g$ means the acceleration of gravity. The value $\theta_{0}$ is the (constant) given temperature of the wall $S_{0}$. Without loss of generality one can suppose that $\theta_{0}=0$ and that $\theta$ is in fact the difference between the physical temperature and $\theta_{0}$. By $p_{a}$ and $\theta_{a}$ we denote the given (constant) pressure and temperature of the ambient air, respectively.

Furthermore, the subsequent notations have been used: $\mathbf{n}$ and $\boldsymbol{\tau}$ are unit vectors normal and tangential to $\Gamma_{1}$ and oriented as $x_{2}, x_{1}$, respectively. By $\mathbf{a} \cdot \mathbf{b}$ we mean the inner product of $\mathbf{a}, \mathbf{b} \in \mathbb{R}^{2}, \nabla=\left(\partial / \partial x_{1}, \partial / \partial x_{2}\right)^{T}$ is the gradient operator, $\nabla p=\operatorname{grad} p, \nabla \cdot \mathbf{v}=\operatorname{div} \mathbf{v}$, $\left.\rho\right|_{\Omega_{m}}=\rho_{m}(m=1,2)$ is the restriction of $\rho$ to $\Omega_{m}$ (analogously for $\mathcal{v}$ and $\lambda$ ) and $\nabla^{2}$ denotes the Laplace operator. By $\mathbf{S}(\mathbf{v})$ we denote the deviatoric stress tensor, that is, a matrix with elements $S_{i j}(\mathbf{v})=\rho v\left(\partial v_{i} / \partial x_{j}+\partial v_{j} / \partial x_{i}\right)(i, j=1,2)$. The symbol $\left.[w]\right|_{\Gamma_{1}}$ denotes the jump of $w$ crossing the free interface $\Gamma_{1}$, that is,

$$
\left.\left[w\left(\mathbf{x}_{0}\right)\right]\right|_{\Gamma_{1}}:=\lim _{\mathbf{y} \rightarrow \mathbf{x}_{0}} w(\mathbf{y})-\lim _{\mathbf{x} \rightarrow \mathbf{x}_{0}} w(\mathbf{x}), \quad\left(\mathbf{x}_{0} \in \Gamma_{1}, \mathbf{y} \in \Omega_{1}, \mathbf{x} \in \Omega_{2}\right),
$$

and the symbol $\left.w\right|_{\Gamma_{1}^{-}}$denotes the limit from below at the interface $\Gamma_{1} ;$ more precisely

$$
\left.w\left(\mathbf{x}_{0}\right)\right|_{\Gamma_{1}^{-}}:=\lim _{\mathbf{y} \rightarrow \mathbf{x}_{0}} w(\mathbf{y}), \quad\left(\mathbf{x}_{0} \in \Gamma_{1}, \mathbf{y} \in \Omega_{1}\right)
$$

Note that the left-hand side of (1.3) 6 (i.e., of the sixth equation in (1.3)) is equal to the curvature $K_{1}\left(x_{1}\right)$ of $\Gamma_{1}$. The same is true for $K_{2}\left(x_{1}\right)$ in case of $\Gamma_{2}$. Furthermore, $(1.3)_{5}$ represents the mathematical expression of the so-called Marangoni convection.

\section{General Solution Technique}

Mathematical problems for the stationary flows of a viscous incompressible fluid with a free boundary were studied by many authors. Numerous references on this topic can be found, 
for example, in the bibliographies of [7-10]. In the analytical investigations $[1-3,5,11]$, the temperature dependence was additionally taken into account. Numerical studies of nonisothermal free boundary problems can be found in the papers $[1,6]$.

For free boundary problems in which the unknown flow domain is unbounded in two directions as in Problem (1.1)-(1.4), a special linearization scheme is necessary (cf. $[7,8]$ and others).

In order to solve such kind of problems-in [7], and independently in [12], an appropriate scheme was proposed based on a linearization of the original problem on a corresponding exact solution in the unperturbed "uniform" flow domain, say: $\Pi=\{\mathrm{x} \in$ $\left.\mathbb{R}^{2}: 0<x_{2}<h_{1} \vee h_{1}<x_{2}<h_{2}\right\}$. The main difference of this scheme from previous applied ones is that on each step of iterations the determination of $\mathbf{v}, p, \theta$ is not separated from the determination of the free boundaries $\Gamma_{i}(i=1,2)$ (i.e., from the determination of the functions $\psi_{i}$ describing $\Gamma_{i}$ ). For Problem (1.1)-(1.4) this scheme can be illustrated by the diagram

$$
\left(\mathbf{v}^{0}, p^{0}, \theta^{0}, \psi_{1}^{0}, \psi_{2}^{0}\right) \longrightarrow\left(\mathbf{v}^{1}, p^{1}, \theta^{1}, \psi_{1}^{1}, \psi_{2}^{1}\right) \longrightarrow \cdots \longrightarrow\left(\mathbf{v}^{m}, p^{m}, \theta^{m}, \psi_{1}^{m}, \psi_{2}^{m}\right) \longrightarrow \cdots
$$

where on each step of iterations the linearized problem is solved in the same "strip-like" domain and the functions $\mathbf{v}, p, \theta$, and $\psi_{i}(i=1,2)$ are determined simultaneously.

A significant part in deriving the correct linearization takes the determination of exact solutions of the nonlinear problems in a "uniform" (not distorted) flow domain. These exact (basic) solutions in the uniform domain $\Pi$ will be calculated in the appendix. They are also important for the numerical flow simulation: they can be used as inlet boundary data in more complicated problems. In [8] the analogous isothermal problem (without any inclusion of temperature) to Problem (1.1)-(1.4) was solved by numerical methods.

\section{Function Spaces}

When studying Problem (1.1)-(1.4), it is useful to work with weighted Sobolev spaces. Let $\Pi_{m}(m=1,2)$ be the strip-like domains

$$
\begin{aligned}
& \Pi_{1}:=\left\{\mathbf{x} \in \mathbb{R}^{2}: 0<x_{2}<h_{1},-\infty<x_{1}<+\infty\right\}, \\
& \Pi_{2}:=\left\{\mathbf{x} \in \mathbb{R}^{2}: h_{1}<x_{2}<h_{2},-\infty<x_{1}<+\infty\right\},
\end{aligned}
$$

and $\Pi=\Pi_{1} \cup \Pi_{2}$ their union. We introduce the space $W_{\beta}^{l, 2}(\Pi)$ of functions $\mathbf{u}$ on $\Pi$ with restrictions $\mathbf{u}^{(m)}=\left.\mathbf{u}\right|_{\Pi_{m}}$ belonging to $W_{\beta}^{l, 2}\left(\Pi_{m}\right)(m=1,2)$ having the finite norms

$$
\left\|\mathbf{u}^{(m)} ; W_{\beta}^{l, 2}\left(\Pi_{m}\right)\right\|=\left\|\mathbf{u}^{(m)} \exp \left(\beta \sqrt{1+x_{1}^{2}}\right) ; W^{l, 2}\left(\Pi_{m}\right)\right\|, \quad(m=1,2)
$$

where $W^{l, 2}\left(\Pi_{m}\right)$ is the usual Sobolev space. The norm in $W_{\beta}^{l, 2}(\Pi)$ is given by

$$
\left\|\mathbf{u} ; W_{\beta}^{l, 2}(\Pi)\right\|=\sum_{m=1}^{2}\left\|\mathbf{u}^{(m)} \exp \left(\beta \sqrt{1+x_{1}^{2}}\right) ; W^{l, 2}\left(\Pi_{m}\right)\right\|
$$


If $\beta>0$, then elements of $W_{\beta}^{l, 2}(\Pi)$ vanish exponentially as $\left|x_{1}\right| \rightarrow \infty$, and if $\beta<0$, then elements $\mathbf{u} \in W_{\beta}^{l, 2}(\Pi)$ might exponentially increase as $\left|x_{1}\right| \rightarrow \infty$.

The spaces $W_{\beta}^{l-1 / 2,2}(\mathbb{R})$ of functions defined on $\mathbb{R}$ can be introduced analogously. Let $S=\left\{x \in \Pi: x_{1} \in \mathbb{R}, x_{2}=h \in\left\{0, h_{1}, h_{2}\right\}\right\}$ be a line. Denote by $W_{\beta}^{l-1 / 2,2}(S)$ the spaces of traces on $S$ of functions from $W_{\beta}^{l, 2}(\Pi)$. Then $W_{\beta}^{l-1 / 2,2}(\mathbb{R})$ coincides with $W_{\beta}^{l-1 / 2,2}(S)$, that is, if $\mathbf{u} \in W_{\beta}^{l-1 / 2,2}(\Pi)$, then $\mathbf{u}(\cdot, h) \in W_{\beta}^{l-1 / 2,2}(\mathbb{R})$.

In the paper the spaces of scalar and vector-valued functions are not distinguished in notations. The norm for vector-valued functions is then the sum of the norms of the corresponding coordinate functions.

\section{Solvability Results}

Problem (1.1)-(1.4) can be handled by the same methods as in $[8,13]$. Let us start with the main result about this problem.

Theorem 4.1. Let $S_{0}=\left\{\mathbf{x} \in \mathbb{R}^{2}: x_{2}=\varepsilon \varphi_{0}\left(x_{1}\right),-\infty<x_{1}<+\infty\right\}, \varphi_{0} \in W_{\beta}^{l+5 / 2,2}(\mathbb{R})$ with $l \geq 0$, and $\beta=\left|\beta_{0}\right| \sin \alpha>0$, where $\alpha$ denotes the slope of the inclined bottom $S_{0}$. Assume that $\alpha$ is sufficiently small. Then there exist positive numbers $\widehat{\varepsilon}, \widehat{r}$ such that for every $\varepsilon \in(0, \widehat{\varepsilon})$. Problem (1.1)-(1.4) has a unique solution $\left(\mathbf{v}, p, \theta, \psi_{1}, \psi_{2}\right)^{T}$. The solution admits the representation

$$
\begin{gathered}
\mathbf{v}(\mathbf{x})=\mathbf{v}^{0}(\mathbf{x})+\varepsilon \mathbf{u}(\mathbf{x}), \quad p(\mathbf{x})=p^{0}(\mathbf{x})+\varepsilon q(\mathbf{x}), \quad \theta(\mathbf{x})=\theta^{0}(\mathbf{x})+\varepsilon \vartheta(\mathbf{x}), \\
\psi_{1}\left(x_{1}\right)=h_{1}+\varepsilon \Psi_{1}\left(x_{1}\right), \quad \psi_{2}\left(x_{1}\right)=h_{2}+\varepsilon \Psi_{2}\left(x_{1}\right),
\end{gathered}
$$

where $\left\{\mathbf{v}^{0}, p^{0}, \theta^{0}\right\}$ are the functions of the basic solution from (A.3)-(A.6), while

$$
\begin{aligned}
\mathbf{U} & :=\left(\mathbf{u}, q, \vartheta, \Psi_{1}, \Psi_{2}\right)^{T} \in\left[W_{\beta}^{l+2,2}(\Pi)\right]^{2} \times W_{\beta}^{l+1,2}(\Pi) \times W_{\beta}^{l+2,2}(\Pi) \times\left[W_{\beta}^{l+5 / 2,2}(\mathbb{R})\right]^{2} \\
& \equiv \Phi_{\beta}^{l, 2} W(\Pi),
\end{aligned}
$$

and the following inequalities hold:

$$
\left\|\mathbf{U} ; \Phi_{\beta}^{l, 2} W(\Pi)\right\| \leq \widehat{r}, \quad \widehat{\varepsilon} \leq \text { const } \cdot \sin ^{2} \alpha .
$$

We would like to present a short sketch of the proof of this theorem by successive approximations. First, the original (perturbed) and unknown flow domain $\Omega$ (cf. Figure 1) is transformed onto the uniform (strip-like) domain $\Pi$. Then, using this transformation mapping, the original flow Problem (1.1)-(1.4) is linearized over the basic solution (A.3)(A.6) (see the appendix) in domain $\Pi$. By $\mathfrak{N}$ we denote the operator of the left-hand side of that linearized auxiliary problem. In virtue of a corresponding theorem for the linear auxiliary problem (see, e.g., [13]), there exists a bounded inverse operator $\mathfrak{N}^{-1}$ such that

$$
\mathfrak{N}^{-1}: \quad \mathcal{R}_{\beta}^{l, 2} W(\Pi) \longmapsto \Phi_{\beta}^{l, 2} W(\Pi),
$$


with $\beta=\left|\beta_{0}\right| \sin \alpha$. Herein $\beta_{0}$ is independent of $\alpha$ and depends on eigenvalues of the operator pencils associated with the corresponding linear problem (cf. [13]). Also, the multidimensional space $\mathcal{R}_{\beta}^{l, 2} W(\Pi)$ to which the right-hand side of the linearized problem belongs is introduced in a similar way as that of the space $\Phi_{\beta}^{l, 2} W(\Pi)$ (see above). Moreover, one can show that there holds the estimate

$$
\left\|\mathfrak{N}^{-1}\right\| \leq \frac{C}{\sin \alpha}
$$

with a constant $C$ being independent of $\alpha$. Therefore, Problem (1.1)-(1.4) is equivalent to the following operator equation in the space $\Phi_{\beta}^{l, 2} W(\Pi)$ :

$$
\mathbf{U}=\mathfrak{N}^{-1} \mathfrak{F}(\mathbf{U}) \equiv \mathfrak{K}(\mathbf{U}),
$$

where $\mathbf{U}=\left(\mathbf{u}, q, \vartheta, \Psi_{1}, \Psi_{2}\right)^{T}$, and $\mathfrak{F}(\mathbf{U})=\left(f_{1}\left(\mathbf{u}, q, \vartheta, \Psi_{1}, \Psi_{2}\right), f_{2}\left(\mathbf{u}, q, \vartheta, \Psi_{1}, \Psi_{2}\right), 0, f_{3}(\mathbf{u}, q, \vartheta\right.$, $\left.\left.\Psi_{1}, \Psi_{2}\right), \ldots\right)^{T}$ denotes the long vector of right-hand side after the linearization. The elements of the right-hand side vector depend on $\varepsilon$ via the transformation mapping of the original flow domain. In order to show the convergence of the successive approximations $\left(\mathbf{u}^{(n)}, q^{(n)}, \vartheta^{(n)}, \Psi_{1}^{(n)}, \Psi_{2}^{(n)}\right)^{T}$, it is sufficient to show that the operator $\mathfrak{K}$ is a contraction mapping in a ball of the space $\Phi_{\beta}^{l, 2} W(\Pi)$ for small values of $\varepsilon$ and $\alpha$.

Note that the corresponding isothermal problem to Problem (1.1)-(1.4) (i.e., without any inclusion of temperature) was analytically examined in details in the papers $[8,13]$. In order to prove Theorem 4.1 in details, one has to repeat and to modify all the investigations from those papers. Since the temperature equation is also nonlinear elliptic there, are not essential changes in the proof. Thus we omit the detailed proof here.

\section{Appendix}

\section{On the Nonisothermal Base Flow}

Let us now investigate the two-fluid flow in the "uniform" two-fluid domain $\Pi$ down the really inclined plane, that is, in the case where $S_{0}$ is replaced by the inclined straight line $S_{*}:=\left\{\mathbf{x}=\left(x_{1}, x_{2}\right) \in \mathbb{R}^{2}: x_{2} \equiv 0,-\infty<x_{1}<+\infty\right\}$. We suppose that the (unknown) free interface and the free surface $\Gamma_{i}$ permit the representations $\Gamma_{i}=\left\{\mathbf{x} \in \mathbb{R}^{2}: x_{2}=\psi_{i}\left(x_{1}\right)=h_{i}=\right.$ const., $\left.-\infty<x_{1}<+\infty\right\}$, and we are looking for stationary unidirectional flows. Such flows fulfil the assumptions

$$
v_{2} \equiv 0, \quad \frac{\partial v_{1}}{\partial x_{1}} \equiv 0, \quad \frac{\partial \theta}{\partial x_{1}} \equiv 0 .
$$

As a consequence we obtain that the pressure gradient downstream is a constant $\partial p / \partial x_{1}=$ $p_{0}=$ const. Since the motion is generated by gravity, only the pressure gradient downstream 
$p_{0}$ must be equal to 0 . Under these assumptions, the nonisothermal Navier-Stokes equations reduce to

$$
\begin{gathered}
-v \rho \nabla^{2} v_{1}=\rho g \sin \alpha, \\
\frac{\partial p}{\partial x_{2}}=-\rho g \cos \alpha, \quad-\lambda \nabla^{2} \theta=0,
\end{gathered}
$$

and the equation of continuity $(1.1)_{2}$ is automatically fulfilled. Problem (1.1)-(1.4) can now be transformed to three systems of equations containing the unknowns $v_{1}\left(x_{2}\right), p\left(x_{2}\right)$, and $\theta\left(x_{2}\right)$, independently. These three systems have the following solutions:

$$
v_{1}^{0}\left(x_{2}\right)=\left\{\begin{array}{cc}
g \sin \alpha\left[-\frac{1}{2 v_{1}} x_{2}^{2}+\left(\frac{h_{2}-h_{1}}{r v_{2}}+\frac{h_{1}}{v_{1}}\right) x_{2}\right], & 0 \leq x_{2} \leq h_{1} \\
g \sin \alpha\left[-\frac{1}{2 v_{2}}\left(h_{2}-x_{2}\right)^{2}+\frac{1}{2 v_{2}}\left(h_{2}-h_{1}\right)^{2}+\frac{1}{2 v_{1}} h_{1}^{2}+\frac{1}{r v_{2}}\left(h_{2}-h_{1}\right) h_{1}\right], & h_{1} \leq x_{2} \leq h_{2} \\
v_{2}^{0}\left(x_{2}\right) \equiv 0, & \text { with } r:=\frac{v_{1} \varrho_{1}}{v_{2} \varrho_{2}},
\end{array}\right.
$$

while

$$
\begin{gathered}
p^{0}\left(x_{2}\right)= \begin{cases}\left(h_{1}-x_{2}\right) \varrho_{1} g \cos \alpha+\left(h_{2}-h_{1}\right) \varrho_{2} g \cos \alpha+p_{a}, & 0 \leq x_{2} \leq h_{1}, \\
\left(h_{2}-x_{2}\right) \varrho_{2} g \cos \alpha+p_{a}, & h_{1} \leq x_{2} \leq h_{2},\end{cases} \\
\theta^{0}\left(x_{2}\right)= \begin{cases}\frac{\theta_{a} \lambda_{2}}{\lambda_{2} h_{1}+\lambda_{1}\left(h_{2}-h_{1}\right)+\lambda_{1} \lambda_{2}} x_{2}, & 0 \leq x_{2} \leq h_{1}, \\
\frac{\theta_{a}\left[\lambda_{1} x_{2}+h_{1}\left(\lambda_{2}-\lambda_{1}\right)\right]}{\lambda_{2} h_{1}+\lambda_{1}\left(h_{2}-h_{1}\right)+\lambda_{1} \lambda_{2}}, & h_{1} \leq x_{2} \leq h_{2} .\end{cases}
\end{gathered}
$$

The so-called fluxes $F_{i}(i=1,2)$ for both liquid layers that are the integrals

$$
F_{1}=\int_{0}^{h_{1}} v_{1}^{(1)}\left(x_{2}\right) \mathrm{d} x_{2}, \quad F_{2}=\int_{h_{1}}^{h_{2}} v_{1}^{(2)}\left(x_{2}\right) \mathrm{d} x_{2}
$$

play an important role in the theoretical proof of the solvability to Problem (1.1)-(1.4). Their values can be calculated as

$$
\begin{gathered}
F_{1}=g \sin \alpha\left[\frac{1}{3 v_{1}} h_{1}^{3}+\frac{1}{2 r v_{2}} h_{1}^{2}\left(h_{2}-h_{1}\right)\right] \\
F_{2}=g \sin \alpha\left[\frac{1}{3 v_{2}}\left(h_{2}-h_{1}\right)^{3}+\left(h_{2}-h_{1}\right)\left(\frac{1}{2 v_{1}} h_{1}^{2}+\frac{1}{r v_{2}} h_{1}\left(h_{2}-h_{1}\right)\right)\right] .
\end{gathered}
$$


Recall that if $\sin \alpha$ is small, then for the fluxes $F_{i}$ the same is true. Furthermore, let us emphasize that solution (A.3)-(A.6) to problem (A.2) is frequently called Nusselt solution of the inclined film flow in the fluid mechanical literature.

\section{Acknowledgment}

The author is grateful for the comments and suggestions of the anonymous referee.

\section{References}

[1] C. Cuvelier and J. M. Driessen, "Thermocapillary free boundaries in crystal growth," Journal of Fluid Mechanics, vol. 169, pp. 1-26, 1986.

[2] P. Ehrhard and S. H. Davis, "Non-isothermal spreading of liquid drops on horizontal plates," Journal of Fluid Mechanics, vol. 229, pp. 365-388, 1991.

[3] V. V. Pukhnachov, "Free boundary problems in the theory of thermocapillary convection," in Free Boundary Problems: Application and Theory, Vol. IV (Maubuisson, 1984), A. Bossavit, A. Damlamian, and M. Fremond, Eds., vol. 121 of Research Notes in Mathematics, pp. 405-415, Pitman, Boston, Mass, USA, 1985.

[4] Y. Shen, G. P. Neitzel, D. F. Jankowski, and H. D. Mittelmann, “Energy stability of thermocapillary convection in a model of the float-zone crystal-growth process," Journal of Fluid Mechanics, vol. 217, pp. 639-660, 1990.

[5] J. Socolowsky, "Existence and uniqueness of the solution to a free boundary-value problem with thermocapillary convection in an unbounded domain," Acta Applicandae Mathematicae, vol. 37, no. 1-2, pp. 181-194, 1994.

[6] J. Socolowsky, "On the numerical solution of heat-conducting multiple-layer coating flows," Lietuvos Matematikos Rinkinys, vol. 38, no. 1, pp. 125-147, 1998, English translation in Lithuanian Mathematical Journal, vol. 1, pp. 98-116, 1998.

[7] S. Nazarov and K. Pileckas, "On noncompact free boundary problems for the plane stationary NavierStokes equations," Journal für die Reine und Angewandte Mathematik, vol. 438, pp. 103-141, 1993.

[8] K. Pileckas and J. Socolowsky, "Viscous two-fluid flows in perturbed unbounded domains," Mathematische Nachrichten, vol. 278, no. 5, pp. 589-623, 2005.

[9] V. A. Solonnikov, "On the Stokes equations in domains with nonsmooth boundaries and on viscous incompressible flow with a free surface," in Nonlinear Partial Differential Equations and Their Applications. College de France Seminar, Vol. III (Paris, 1980/1981), vol. 70 of Research Notes in Mathematics, pp. 340-423, Pitman, Boston, Mass, USA, 1982.

[10] V. A. Solonnikov, "Solvability of a problem of the flow of a viscous incompressible fluid into an infinite open basin," Trudy Matematicheskogo Instituta imeni V. A. Steklova, vol. 179, pp. 174-202, 1988 (Russian), English translation in Proceedings of the Steklov Institute of Mathematics, vol. 179, no. 2, pp. 193-226, 1989.

[11] M. V. Lagunova, "Solvability of a plane problem of thermocapillary convection," in Linear and Nonlinear Boundary Value Problems. Spectral Theory, vol. 10 of Probl. Mat. Anal., pp. 33-47, Leningrad University, Leningrad, Russia, 1986.

[12] F. Abergel and J. L. Bona, "A mathematical theory for viscous, free-surface flows over a perturbed plane," Archive for Rational Mechanics and Analysis, vol. 118, no. 1, pp. 71-93, 1992.

[13] K. Pileckas and J. Socolowsky, "Analysis of two linearized problems modeling viscous two-layer flows," Mathematische Nachrichten, vol. 245, pp. 129-166, 2002. 


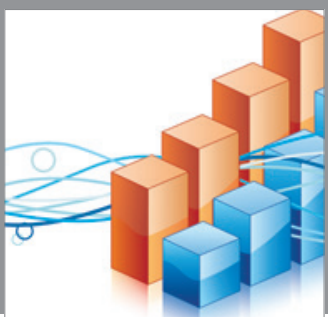

Advances in

Operations Research

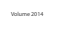

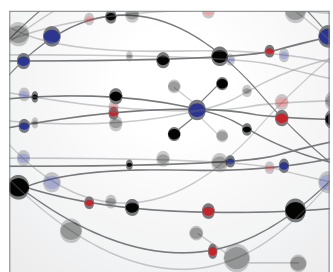

\section{The Scientific} World Journal
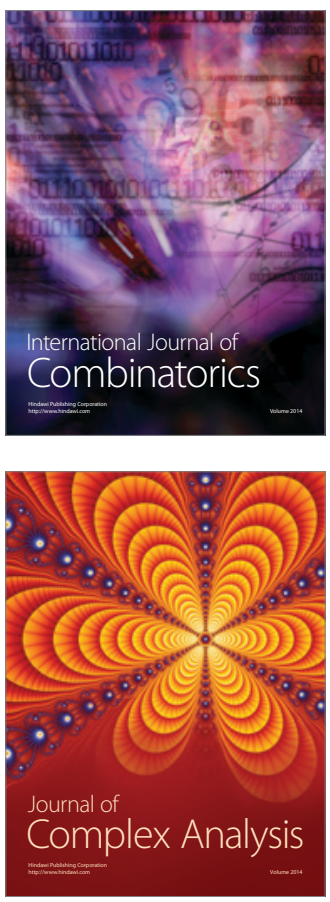

International Journal of

Mathematics and

Mathematical

Sciences
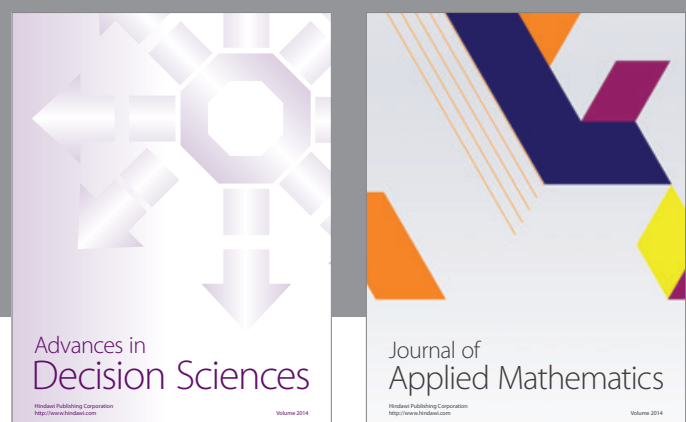

Journal of

Applied Mathematics
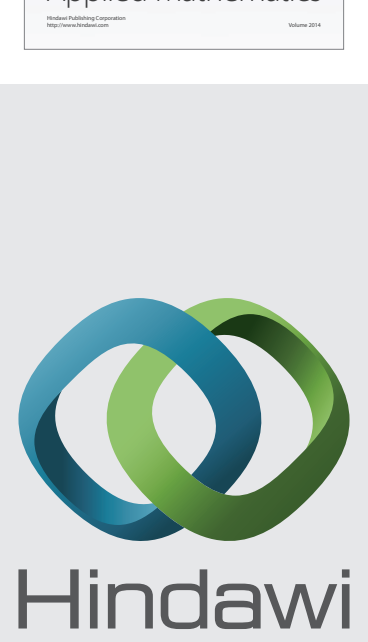

Submit your manuscripts at http://www.hindawi.com
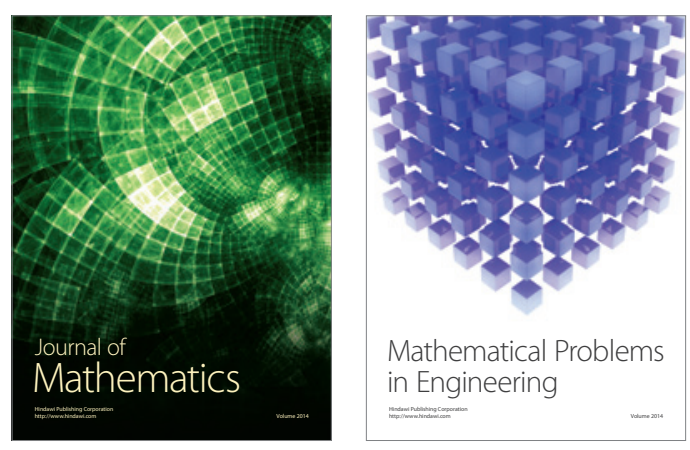

Mathematical Problems in Engineering
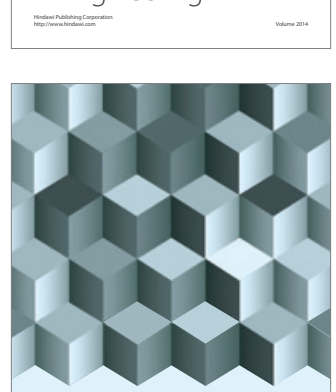

Journal of

Function Spaces
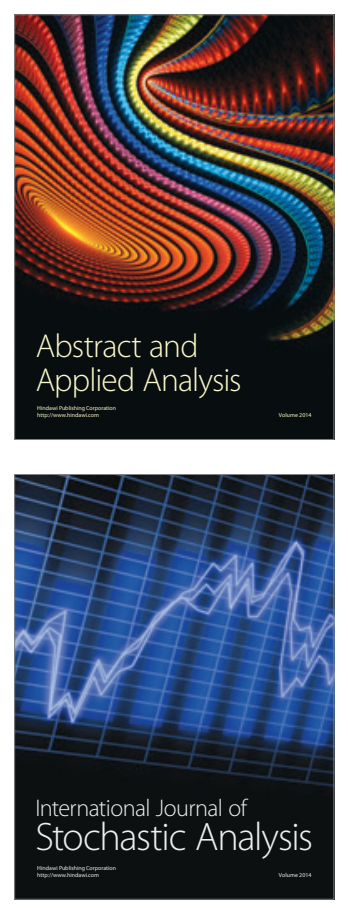

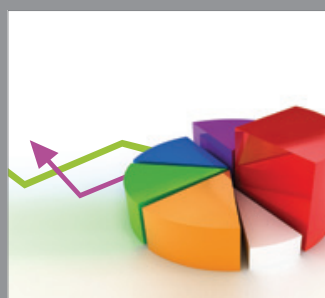

ournal of

Probability and Statistics

Promensencen
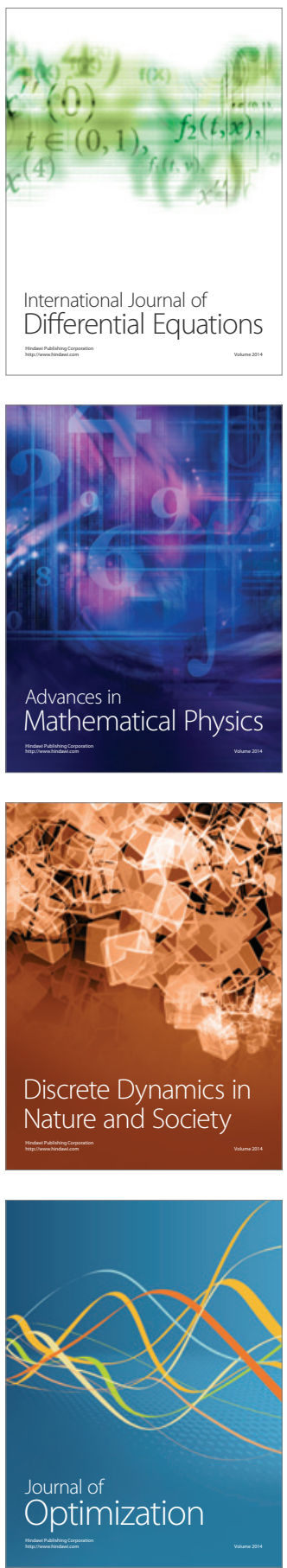